\title{
Immer wieder 2001 - Anmerkungen zur aktuellen rentenpolitischen Debatte
}

\author{
FLORIAN BLANK
}

Die Alterssicherung ist ein Dauerbrenner in der deutschen Politik. Nachdem im Jahr 2011 viel über die „Riester-Rente“ diskutiert wurde, kreist die gegenwärtige Debatte vor allem um die Gesetzliche Rentenversicherung (GRV) und ihre (erwarteten) Leistungen. Im Zentrum standen zunächst Reformvorschläge der Bundesministerin für Arbeit und Soziales, Ursula von der Leyen, die im Rahmen des „Rentendialogs“ vorgestellt wurden. Anfang November hat sich der Koalitionsausschuss auf „Verbesserungen für eine Lebensleistungsrente" verständigt. Befeuert wird die Debatte zudem durch die SPD: Deren Vorsitzender Sigmar Gabriel hat ebenfalls Reformvorschläge unterbreitet, die schließlich zu einem Beschluss des Parteivorstands, einem Antrag an den Parteikonvent im November 2012, führten.

Die Vorschläge betreffen eine ganze Reihe unterschiedlicher Aspekte. Zentraler Baustein des Vorschlags des Bundesministeriums für Arbeit und Soziales (BMAS) ist die „Zuschussrente“, durch die für bestimmte langjährig Versicherte Renten oberhalb der Grundsicherung im Alter ermöglicht werden sollen. Dieses Ziel ist auch der "Lebensleistungsrente“ zu eigen. Der Beschluss des SPD-Parteivorstandes sieht u.a. die Einführung einer „Solidarrente“ vor.

Ich möchte im Folgenden verdeutlichen, dass diese Reformvorschläge die Weichenstellungen der Rentenreform von 2001 bestätigen - die Politik hat die Kernprinzipien dieser Reform als leitende Norm internalisiert. ${ }^{2}$ Dabei konzentriere ich mich auf die Vorschläge des BMAS und des SPD-Parteivorstands. Die knappen Ausführungen zur Lebensleistungsrente des Koalitionsausschusses fügen sich in die Debatte ein, lassen aber noch Fragen offen. Ursula von der Leyen kündigte allerdings an, dass ein Gesetzentwurf zur Bekämpfung der Altersarmut an die Beschlüsse angepasst werden solle. Die Bestätigung der vergangenen Weichenstellungen betrifft unabhängig von den jeweiligen Details m. E. vor allem drei Punkte: die Leistungsfähigkeit der GRV, die Aktivierung der Bürgerinnen und Bürger auch in der Rentenpolitik und die Verteilung der Finanzierung zwischen Arbeitnehmern und Arbeitgebern.

\section{Das Problem niedriger Renten}

Die Vorschläge zur Zuschussrente und Solidarrente wie auch zur Lebensleistungsrente sollen auf absehbar niedrige Renten reagieren. Als Problem gelten geringe Ansprüche gegenüber der Rentenversicherung, die sich aus Arbeitsmarktentwicklungen, insbesondere aus geringen Löhnen ergeben: Bei einer langen Versicherungsdauer sollen geringe Ansprüche an die Rentenversicherung aufgewertet werden.

Diese Ansätze zielen nicht auf die Bekämpfung von Armut im Alter als Lebenslage, sondern auf eine spezielle Gruppe von „deserving poor“.
Diese haben durch ihre Vorleistungen bewiesen, dass sie einer weiteren Unterstützung würdig sind: Sie sind schon lange ins Sozialversicherungssystem integriert, werden dort aber unzureichend versorgt. Als akzeptable Vorleistungen zählen Erwerbsarbeit (bzw. Bereitschaft zur Erwerbsarbeit) und Familienarbeit, die in der letzten Fassung der Zuschussrente sogar deutlich höheres Gewicht als die Erwerbsarbeit erhielt. In unterschiedlichem Maße sollen bei den neuen Leistungen an diese Gruppe auch andere Einkommensquellen angerechnet, also Bedürftigkeiten geprüft werden.

Das Sinken des Rentenniveaus insgesamt bleibt außen vor. ${ }^{3}$ Implizit wird davon ausgegangen, dass die regelmäßige Lebensstandardsicherung weiter im sogenannten Drei-Säulen-Modell erzielt werden kann (dazu unten mehr). Die Reformpläne reagieren auf den vergleichsweise geringen Wert individueller Ansprüche, wobei der Vergleichsmaßstab das Grundsicherungssystem ist. Dessen Leistungen und Zugangsmodus gelten als für Leistungsträger unwürdig (für diejenigen, die ihren gesellschaftlichen Nutzen nicht bewiesen haben, aber als adäquat). ${ }^{\oplus}$

Für diesen Fokus auf Geringverdiener gibt es gute Gründe und an ernst zu nehmenden Warnungen vor einer Rückkehr der Altersarmut mangelt es im Übrigen auch nicht. ${ }^{\ominus}$ Verschiedene Lösungsvorschläge, wie etwa auch die Fortfüh rung der Rente nach Mindestentgeltpunkten,
Zum Vorschlag des BMAS s. http://www.bmas. de/SharedDocs/Downloads/DE/PDF-Pressemitteilungen/rentendialog-gesamt-pdf.pdf? blob=publicationFile; zu den Beschlüssen des Koalitionsausschuss http://www.cdu.de/doc/ pdfc/121105-Koalitionsausschuss.pdf; zum Beschluss des SPD-Parteivorstands http://www. spd.de/linkableblob/76890/data/20120924_rente_ pv.pdf. Die Arbeit an diesem Beitrag wurde am 5.11.2012 abgeschlossen.

2 So heißt es auch im Beschluss des SPD-Parteivorstandes, dass die SPD die von ihr mitgetragenen Rentenreformen nicht infrage stellt.
3 Der SPD-Vorstand hat jedoch den Vorschlag der nordrhein-westfälischen SPD, das aktuelle Niveau zu sichern, „zustimmend diskutiert".

(4) Die Schaffung der Grundsicherung im Alter und bei Erwerbsminderung war übrigens als Ergänzung der Sozialhilfe damit begründet worden, dass "verschämte Altersarmut" verhindert werden sollte, aber mehr noch: „Ferner soll für die Zukunft vorbeugend verhindert werden, dass die Altersarmut ansteigt. Eine solche Entwicklung kann aus vielfältigen und heute in ihren Auswirkungen noch nicht einschätzbaren Ursachen, wie beispielsweise Brüche in den Er- werbsbiographien oder langfristige Folgen der Arbeitslosigkeit, nicht ausgeschlossen werden" (BT-Drs. 14/4595, S. 43; Gesetzentwurf der Bundesregierung zum Altersvermögensgesetz). In dem Verweis auf den Arbeitsmarkt ähnelt die damalige Begründung der heutigen Debatte. Allerdings wurde in diesem Entwurf offensichtlich unterstellt, dass die Grundsicherung allein schon vor Armut schützt.

(5) http://www.boeckler.de/14_40971.htm. 
sind mithin ernsthaft zu diskutieren. Allerdings verstellt der Fokus auf Anbauten am unteren Geschoss des Rentensystems den Blick darauf, was in den mittleren oder oberen Stockwerken geschieht. Eine einfache Ableitung geringer Renten aus geringen Ansprüchen gegenüber der Rentenversicherung infolge der Arbeitsmarktentwicklung greift zu kurz. Die Renten werden in die Zange genommen. Neben geringe Ansprüche tritt die geringere Bewertung aller Ansprüche. Das heißt aber, dass sich auch für Beschäftigte mit mittleren Einkommen zunehmend die Frage stellt, ob sie nicht auch Kandidaten für eine neue Mindestsicherung werden: 2011 lag der durchschnittliche Rentenzahlbetrag im Rentenzugang bei Altersrenten nach Abzug der Sozialabgaben im Westen bei $868 €$ (Männer) bzw. $487 €$ (Frauen), im Osten bei $867 €$ (Männer) bzw. $681 €$ (Frauen). ${ }^{\odot}$ Des ungeachtet suggerieren die genannten Vorschläge jedoch, dass das Alterssicherungssystem insgesamt problemfrei arbeitet und nur besonders unglückliche Lebensläufe eines Ausgleichs bedürfen.

\section{Die Verstetigung der Aktivierung}

Das Drei-Säulen-Modell aus GRV, betrieblicher Altersversorgung (bAV) und privater Vorsorge mitsamt seinen gesellschaftspolitischen Implikationen (Aktivierung, Eigenvorsorge) ist und bleibt das gängige Paradigma; eine Rückkehr zur Rentenversicherung als allein ausreichender Form der Sicherung kann sich in der aktuellen Diskussion nicht durchsetzen. Bürgerinnen und Bürger sind gehalten, eigenverantwortlich Entscheidungen für ihre Alterssicherung zu treffen. Die öffentliche Förderung dieser Eigenvorsorge hat nicht nur sozialpolitisch-unterstützenden Charakter, sondern dient auch der Animation zur aktiven Gestaltung der eigenen Sicherung.

Vom SPD-Parteivorstand wird der Ausbau der betrieblichen Altersversorgung gefordert, wie auch Verbesserungen bei der "Riester-Rente“. Das BMAS sieht bei der „Riester-Rente“ ebenso Handlungsbedarf, transportierte aber die Aktivierungslogik im Rahmen der Zuschussrente zurück in die Rentenversicherung: Als eine Voraussetzung für den Erhalt der öffentlichen $\mathrm{Zu}$ schussrente war die Nutzung der zusätzlichen Altersvorsorge vorgesehen. Dieser Faden wird auch bei der Lebensleistungsrente aufgenommen. Das ist auf den ersten Blick sogar schlüssig. Denn in einem Gesamtsicherungssystem, das bei der Leistungserbringung nicht auf die Rentenversicherung allein setzt, scheint der Verweis auf eben dieses Gesamtsystem auch bei der Leistungsvoraussetzung für die Gewährung einer Mindest- leistung stimmig. Wenn die Bürgerinnen und Bürger vorsorgen sollen, um das Ziel der Lebensstandardsicherung zu erreichen, warum sollte man dann das Ziel Armutsvermeidung nicht genau so angehen?

Allerdings lässt sich diese Konstellation auch anders darstellen und ist dann nur noch bizarr zu nennen. Die Pläne bedeuten nämlich, dass erst durch Zahlungen an die Versicherungsindustrie oder Banken erweiterte Leistungsansprüche gegenüber der öffentlich-rechtlichen Rentenversicherung erworben werden. Während nun aber das Drei-Säulen-Modell zum Türsteher für eine öffentliche Sozialleistung befördert wird, soll die Höhe der Leistungen aus diesem System bei der Gewährung und Bemessung der Zuschussrente nicht berücksichtigt werden. Es ist vom Standpunkt der seit 2001 geltenden Systemlogik schon erstaunlich, dass die Leistungen aus der gesetzlichen Rentenversicherung im Mittelpunkt stehen, bAV und private Vorsorge im Unterschied zu anderen Einkommensarten aber nicht angerechnet werden. Das Drei-Säulen-Modell wird hier leistungsseitig nicht in die Pflicht genommen.

In diesen Vorschlägen offenbart sich übrigens auch ein struktureller Unterschied zwischen öffentlicher Rentenversicherung und privater Vorsorge: In der öffentlichen Rentenversicherung besteht die Möglichkeit auch einer nachträglichen Neubewertung von Ansprüchen, das heißt einer sozialpolitischen Steuerung aufgrund einer Auseinandersetzung über den Wert von Vorleistungen. In der privaten Vorsorge ist ein ausgleichender Eingriff nur ex ante durch die Förderung machbar - der Ausgleich zwischen privaten Konten und damit letztlich den Ergebnissen von Transaktionen auf Finanzmärkten ist im Nachhinein schwer denkbar und allenfalls über das Steuerrecht zu regeln.

Das BMAS und auch die SPD rücken dagegen in anderen Bereichen vom Mantra der Eigenverantwortung ab und betonen den Schutzcharakter der öffentlichen Sozialpolitik wieder etwas stärker: bei der Absicherung Selbstständiger wie auch bei der bAV (der SPD-Parteivorstand fordert ein Arbeitgeber-Obligatorium, d.h. Arbeitgeber müssen die Entgeltumwandlung anbieten, sofern der Arbeitnehmer nicht darauf verzichtet.). Den Schritt zu einer regelmäßigen und einheitlichen Absicherung möchten die Akteure jedoch beide nicht so ganz gehen. In den Konzepten sind Wahlmöglichkeiten vorgesehen, jedoch können sie vor der normativen Folie einer flächendeckenden Absicherung zumindest als Schritte in diese Richtung gewertet werden.

\section{Die Verteilungsfrage}

In der Debatte um Altersarmut und die Sicherung von Selbstständigen, der Diskussion um den Beitragssatz und die Probleme, die Verbraucher mit der „Riester-Rente“ haben, wird in den Vorschlägen ein zentraler Baustein der Rentereform 2001 nicht behandelt: die Abkehr von der paritätischen Finanzierung im Gesamtsystem. Zur Erinnerung: Mit dem Ziel der Stabilisierung der (paritätisch getragenen) Beiträge zur GRV wurde 2001 das Leistungsziel Lebensstandardsicherung auf das Alterssicherungssystem aus GRV, bAV und privater Vorsorge übertragen. $\mathrm{Zu}$ dessen Finanzierung sind aber neben den Sozialversicherungsbeiträgen weitere Beiträge in Höhe von $4 \%$ des Bruttoeinkommens einschließlich der öffentlichen Zulagen notwendig (so die Annahme der damaligen und aller folgenden Bundesregierungen). Diese Beiträge müssen zunächst von den Beschäftigten und ihren Familien getragen werden, unterstützt durch öffentliche Zuschüsse und Steuer- bzw. Sozialabgabenbegünstigungen. Die Arbeitgeber sind also aus dem Spiel.

In der bAV stellt sich die Lage zwar etwas besser dar. Hier wurden teils beachtliche Tarifverträge geschlossen, durch die die Arbeitgeber an der Finanzierung der Alterssicherung auch neben der GRV beteiligt bleiben. Des ungeachtet ist aber festzuhalten, dass so die Verantwortung für die Finanzierung auf die Sozialpartner abgeschoben wurde und die Verteilung der Kosten in dieser Säule nun von deren Verhandlungsmacht und -geschick wie auch der allgemeinen wirtschaftlichen Situation einer Branche abhängt (ganz zu schweigen von der Tarifbindung der Betriebe). In den aktuellen Vorschlägen wird diese verteilungspolitische Weichenstellung nicht korrigiert.

\section{Was ist gut?}

In der laufenden Diskussion bleibt das Mischsystem aus gesetzlicher Rentenversicherung und anderen Sicherungswegen zentraler Bezugspunkt. Eine generelle Niveauverbesserung unter Inkaufnahme verteilungspolitischer Konflikte scheint nicht mehrheitsfähig.

Bleibt also alles beim Neuen? Nach der Kritik erstaunt vielleicht die etwas versöhnlichere Note zum Schluss, doch scheint - ungeachtet der Mo-

6 Deutsche Rentenversicherung Bund (2012): Rentenversicherung in Zahlen 2012, S. 52-55. 
tive von Parteien, Ministerium und Einzelpersonen - eine Beobachtung erfreulich: Es wird überhaupt wieder über Leistungen gestritten. Die heilige Kuh „Beitragssatzstabilität“ erfreut sich zwar guter Gesundheit (zeitgleich mit der Debatte um Leistungsverbesserungen wird durch die Koalition die Senkung des Beitragssatzes zur Rentenversicherung durchgesetzt). Die Frage, welche Leistungen denn sinnvoll und notwendig sind, hat aber zumindest im Zuge der Debatte um Altersarmut eine vorsichtige Rückkehr auch bei den Akteuren erlebt, die die Rentenpolitik seit den 1990er Jahren maßgeblich mitgestaltet haben. Die Annahme der Jahre 2001ff., dass das Gesamtsys- tem der Alterssicherung für alle Bürgerinnen und Bürger (oder zumindest für alle Arbeitnehmerinnen und Arbeitnehmer) die Lebensstandardsicherung garantiert und der Arbeitsmarkt das seine zu einem Leben im Alter im relativen Wohlstand beiträgt, ist damit zumindest relativiert. Wenn das als ein Impuls zu einer neuen Sozialund Arbeitsmarktpolitik dienen kann, die die gröbsten Verwerfungen der letzten Jahre behebt, und zu einer allgemeinen sozialpolitischen Debatte, welche Leistungen in der gesetzlichen Rentenversicherung denn für wen als fair angesehen werden, dann ist noch wenig Materielles, aber immerhin etwas gewonnen.

\section{AUTOR}

FLORIAN BLANK, Dr., ist Wissenschaftler im Wirtschafts- und Sozialwissenschaftlichen Institut (WSI) in der Hans-Böckler-Stiftung. Arbeitsschwerpunkt: Sozialpolitik.

florian-blank@boeckler.de 